\title{
Very fast relaxation in polycarbonate glass
}

\author{
L. Saviot, E. Duval \\ Laboratoire de Physico-Chimie des Matériaux Luminescents, UMR-CNRS 5620, Université Lyon \\ I , 69622 Villeurbanne Cedex, France \\ J. F. Jal \\ Département de Physique des Matériaux, UMR-CNRS 5586, Université Lyon I, 69622 \\ Villeurbanne Cedex, France \\ A. J. Dianoux \\ Institut Laue-Langevin, F38042 Grenoble Cedex, France
}

(May 6, 2019)

\begin{abstract}
Relaxations in amorphous bis-phenol A polycarbonate are studied by neutron scattering, as a function of temperature below the glass transition. Two different processes are observed. One is very fast, with a characteristic time $(\sim 0.3 p s)$, that is independent of temperature and momentum transfer. Conversely the other is slower, with a time, which is dependent on temperature and momentum transfer. The very fast localized anharmonic motion is interpreted by the overdamping of low-frequency vibrational modes, by nearby dynamic holes. The slower relaxation is thermally activated and momentum transfer dependent. It corresponds to molecular group motions and possibly to the short-time regime of the segmental relaxation.
\end{abstract}

PACS numbers: 6141.+e, 61.12.Ex, 64.70.Pf 


\section{INTRODUCTION}

Anharmonic motions are observed in glasses, and in particular in amorphous polymers, by inelastic neutron scattering and inelastic light scattering down to temperatures much lower than the temperature of glass transition, $T_{g}$. These non-harmonic motions appear in the non-linear temperature dependence of the mean square displacement, and are responsible for the quasielastic scattering. In the case of amorphous poly(methyl methacrylate) (PMMA) it was deduced, from neutron or light scattering measurements, that the high-frequency tail of the quasielastic scattering is Lorentzian, the half width at half maximum (HWHM) of the corresponding Lorentz curve being close to $1 \mathrm{meVt}$ 3. Furthermore the HWHM was found independent of the momentum transfer and very weakly dependent on temperature. On the other hand the intensity of quasielastic scattering was shown to be strongly dependent on temperature. Such characteristics indicate that the quasielastic scattering is due to localized and weakly thermally activated relaxations of molecular groups. These localized motions are released by a slower relaxation, that is thermally activated. This last point was confirmed by the comparison of the quasielastic light scattering (QELS) with the volume fraction of

dynamic holes, which was determined by positron annihilation lifetime spectroscopy $\mathbf{A}^{\mathrm{B}}$. It was shown that the thermal behavior of the QELS, that is proportional to the number of relaxing units, and of the hole volume fraction, are identicall 1 . From this comparison it is clear that the temperature dependence of quasielastic intensity comes from the one of the number of relaxing units. Similar conclusions were deduced from measurements on amorphous bis-phenol A polycarbonate (PC)6.

The study of the relaxational motions by inelastic or quasielastic neutron scattering allows us to analyse the dynamics of relaxation in PC from about 0.1 up to 10 picoseconds. The corresponding neutron measurements are presented in this paper. The presence of very fast localized anharmonic motions, with a characteristic time close to $0.3 \mathrm{ps,}$ was confirmed. Similar very fast relaxations were observed in the ortho-terphenyl glass by depolarized light

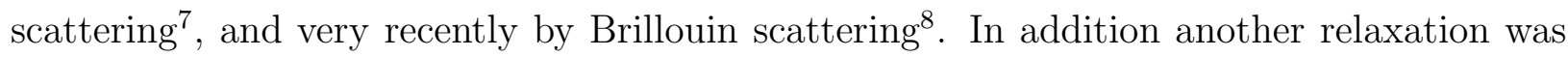


observed, with a time, which is dependent on temperature and momentum transfer. In a recent paper Colmenero and Arbe 9 described the relaxation in polymer glasses, such as PC, at temperature larger than a critical one, $T_{f}$, by a model similar to the one used for the segmental $\alpha$-relaxation in the supercooled state 10 . According to this model, the relaxation is Debye-like up to a critical time $\tau_{c}$, and becomes slower and non-exponential at longer times. The slower relaxation, that is observed in this work, could correspond partially to the segmental one in the Debye-like regime.

In the first part of this paper the experimental results are presented. It will be shown that the experimental neutron scattering intensities, which are due to relaxations, can be fitted by two exponential decays. The experimental parameters are analyzed and discussed in the second part.

\section{EXPERIMENT}

The PC specimen was purchased from Bayer. The number average molecular weight is $15600 \mathrm{~mol}^{-1}$ with a polydispersity index of 1.85 . The glass transition temperature is around $420 \mathrm{~K}$, as determined by differential scanning calorimetry. The thickness of the samples was equal to $0.29 \mathrm{~mm}$.

The inelastic neutron spectra were recorded on the time-of-flight intrument IN6 at the ILL, Grenoble. The wavelength of the incident neutrons was equal to $5.12 \AA^{-1}$ resulting in an elastic energy resolution (FWHM) of $80 \mu \mathrm{eV}$ and an elastic momentum transfer range

extending from $Q=0.22 \AA^{-1}$ to $Q=2.06 \AA^{-1}$. The spectra were taken in the temperature interval 15-390 K, using a helium cryofurnace. The scattering cross-sections were obtained after the usual standard calibrations by means of the vanadium runs and removal of the empty-can contributions. On the basis that PC mainly yields incoherent scattering (as was experimentally checked), we calculated the inelastic neutron scattering intensity and, then, the total density of states through the use of an iterative procedure described elsewhere 11 . 


\section{EXPERIMENTAL RESULTS}

To show the presence of anharmonic or relaxational motions at $T<T_{g}$, the mean square displacement (MSD), $\left\langle u^{2}\right\rangle$, was deduced from the Debye-Waller factor, $e^{-2 W(Q)}$, where $2 W(Q)=Q^{2}\left\langle u^{2}\right\rangle / 3$, which was determined by plotting the normalized elastic scattering intensity, $S(Q, \omega=0, T) / S(Q, \omega=0,0)$, versus $Q^{2}$, at different temperatures. The logarithm of the normalized intensity is perfectly $Q^{2}$ linear in the explored Q-range $\left(0.2-1.9 \AA^{-1}\right)$. However, as for other polymeric glasses, it was observed that the different straight lines do not converge to a same point at $Q=0$. This is due to the competition between the elastic and the inelastic multiple scatterings 12 : the elastic multiple scattering events are dominating at low temperature, and are gradually replaced by inelastic scattering events with increasing temperature. This defect of elastic scattering at $Q=0$, that is due to multiple scattering, was very recently confirmed by Wuttke13. But, as shown by this author, it has a negligible effect on the slope of straight lines in the considered Q-range and for the thickness (0.29 $\mathrm{mm}$ ) of the experimented samples. The MSD, $\left\langle u^{2}\right\rangle$, is plotted against $\mathrm{T}$ in Figure-1. It is observed that $\left\langle u^{2}\right\rangle$ becomes non-linearly temperature dependent from a temperature slightly higher than $50 \mathrm{~K}$, showing the presence of anharmonic motions from this temperature. This is confirmed by the vibrational density of states (VDOS), which increases with temperature at low energy, from the same temperature 14.

Figure-2a and -2b show the Debye-Waller corrected $S(Q, \omega)$ scattering intensities, respectively at different temperatures for $Q=1.9 \AA^{-1}$ ) and at $T=390 \mathrm{~K}$ for different Q. The inelastic scattering by harmonic modes can be observed even at $30 \mathrm{~K}$. On the other hand, the effect of anharmonic motions is clearly present from $T=220 \mathrm{~K}$. Figure-2b shows that it increases with Q. In order to obtain more informations about the anharmonic or relaxational motions, we determined a time-dependent scattering intensity, $S_{n h}(Q, t)$, which is independent of the harmonic vibrations, by taking the Fourier transform of $S(Q, \omega)$, and

using a procedure similar to the one described by Colmenero et al 10 . In fact $S(Q, \omega)$ is the scattering intensity at constant angle, Q being the momentum transfer for elastic scattering. 
The actual momentum transfer is larger than $\mathrm{Q}$ at the highest frequencies of the considered spectral range. But this variation is without serious consequence, since the fastest decay will be found to be Q-independent. $S(Q, \omega)$ is the convolution product of $S_{n h}(Q, \omega)$ by the harmonic vibration scattering intensity, $S_{h}(Q, \omega)$, and by the instrumental resolution, $R(Q, \omega):$

$$
S(Q, \omega)=S_{n h}(Q, \omega) \otimes S_{h}(Q, \omega) \otimes R(Q, \omega)
$$

The product $S_{h}(Q, \omega) \otimes R(Q, \omega)$ at a temperature $\mathrm{T}$ is given by the experimental scattering intensity at a low temperature, at which the neutron scattering from relaxational motions is negligible, corrected by the Bose and Debye-Waller factors for the temperature T. It was controlled that at $30 \mathrm{~K}$ no relaxation appears in the neutron scattering (Figures 1 and 2). However one observed that there exists, from a temperature of $100 \mathrm{~K}$, a significant softening: An identical relative decrease of the transverse sound velocity, that was observed by Brillouin scattering, and of the Raman peak position or of the frequencies of low-frequency vibrational modes was determined 1 . At $T=400 \mathrm{~K}$ this relative decrease is close to 0.7. To take into account the softening at a temperature T, $S(Q, \omega)$ at $T=30 K$ was divided by the Bose factor at this temperature; in a second step the frequencies were renormalized by the relative decrease coefficient of the vibrational low frequencies; and then the obtained structure factor was multiplied by the Bose factor at temperature $\mathrm{T}$. The Fourier transforms were performed through the real one-dimensional tranform provided by version 2 of the freely available FFTW library (Massachusetts Institute of Technology - http://www.fftw.org/). The $S(Q, \omega)$ data were taken up to $10 \mathrm{meV}$ before performing the fast Fourier transform in order to do it over the same interval for all temperatures. Taking into account this spectral interval and the instrumental resolution a fairly good confidence can be given to the Fourier transforms from t=0 to t $=10$ ps. $S_{n h}(Q, t)$, at temperature $\mathrm{T}$, was then obtained by dividing the Fourier transform $S(Q, t)$ by the Fourier transform $S_{h}(Q, t) \cdot R(Q, t)$ of $S_{h}(Q, \omega) \otimes R(Q, \omega)$ obtained in this way at temperature $\mathrm{T}$. The relative intensity $S_{n h}(Q, t) / S_{n h}(Q, 0)$ is plotted against the time $t$ for different temperatures and $Q=1.9 \AA^{-1}$ in Figure-3, and for different 
momentum transfers and $T=390 K$ in Figure- 4 .

Different mathematical expressions, having a physical meaning, were tested to fit $S_{n h}(Q, t)$. Up to a time $\mathrm{t}=10 \mathrm{ps}$, that is limited by the instrumental resolution, the fits, which are unambiguously the best ones (Figure 3 and 4), were obtained by two exponentials decays:

$$
S_{n h}(Q, t)=\left((1-\rho)+\rho \exp -t / \tau_{1}\right) \exp -t / \tau_{2}
$$

In this expression $\rho \leq 1$. It is emphasized that (3.2) is considered to be valid only at short times $\left(t<\tau_{2}\right)$. The time $\tau_{1}$ is approximately equal to $0.3 p s$ and very weakly dependent on temperature and momentum transfer. The fitting parameters are collected in Table- 1 . The time $\tau_{2}$ decreases with $Q$ and $T\left(\tau_{2} \approx 60 p s\right.$ at $T=390 K$ and $\left.Q=1.9 \AA^{-1}\right)$. It is likely that, at the considered longest times and highest temperatures, the decays can be fitted by a stretched exponential or a distribution of relaxation times. On the other hand, as suggested by Novikov 16 , it is not impossible that the decays, in the 0-10 ps interval, may be fitted by a peculiar distribution of relaxation times. However in this case the analysis of the experimental data is not so straightforward, as it is possible tofit with different sets of parameters.

\section{DISCUSSION}

We will discuss principally the very fast anharmonic motion (symbolized by VFAM), that is characterized by the sub-picosecond time $\tau_{1}$. At first it is noticed that the nonlinearly temperature dependent MSD increases from a temperature much lower than $T_{g}$. It is likely that, at temperatures higher than $120 \mathrm{~K}$, the pure methyl-group rotation and the $\pi-$ flip motions of phenylene contribute to this MSD. It is important to be sure that such molecular motions do not contribute to the VFAM. The activation energy for the methylgroup rotation is weaker than the one for $\pi-$ flip motion: From nuclear magnetic resonance,

they are respectively $15-20 \mathrm{~kJ} / \mathrm{mole}=$, and $30-50 \mathrm{~kJ} / \mathrm{mole}{ }^{18}$. The possible contribution of the 
methyl-group rotation is larger than the one of the phenylene $\pi-$ flip in the $0.2-10$ ps time interval. A broad maximum in the VDOS was observed at $37 \mathrm{meV}$, that corresponds to the methyl-group libration, i.e., to the attempt frequency for rotation of about $10^{-13} s^{-1}$. At the highest temperature of observation, $390 \mathrm{~K}$, the lowest time of rotation, that is obtained from these values of activation energy and attempt frequency, is 10 ps. Consequently, the very short time $\tau_{1}(\simeq 0.3 \mathrm{ps})$, that is approximately temperature independent, cannot correspond to the methyl-group rotation or phenylene $\pi-$ flip.

The VFAM is relatively well characterized. Its decay time $\tau_{1}$ is approximately independent of temperature $T$, and of momentum transfer $Q$ (Table-1). On the other hand the part $\rho$ of $S_{n h}(Q, t)$, due to the VFAM, increases with $T$ and a little with $Q$ (Table- 1 ). The weak Q-dependence of $\rho$ comes probably from a component of rotation in the VFAM 19 . With regard to the temperature dependence, it is interesting to compare $\rho$ to the volume fraction of the dynamical holes determined by positron annihilation lifetime spectroscopy (PALS). From the principle of the technique, the PALS lifetime of the dynamic holes, which have a size close to $0.3 \mathrm{~nm}$, is longer than $2 \mathrm{~ns} 20$, i.e much longer than $\tau_{1}$. It corresponds to the long lifetime $\tau_{3}$ of the ortho-positronium (o-Ps). In consequence the lifetime of the dynamic holes can be considered as infinite for the VFAM. In Figure-5 the ratio $\rho$ is plotted against the dynamic hole volume fraction, $\Delta F_{h}$, that was measured by PALS20. $\Delta F_{h}$ at a temperature T was determined by subtracting the hole volume fraction, that was obtained by extrapolation at $T=0 K$, from the total hole volume fraction at the temperature T. Figure-5 shows a remarkable proportionality between $\rho$ and $\Delta F_{h}$ in the $170-390 \mathrm{~K}$ temperature interval. It is clear that the temperature dependence of $\rho$ corresponds to that of the part of the polymeric glass, which participates to the VFAM.

A proportionality between $\Delta F_{h}$ and the QELS intensity of PMMA and of PC, respectively, was recently observed 15 . From the behavior of the VFAM similar to the one of the QELS, as compared to the $\Delta F_{h}$, it would be deduced that the VFAM observed by neutron scattering corresponds to the QELS. It does not seem to be the case, because, principally, the time $\tau_{1}$ is more than three times shorter than the one measured, and theoretically jus- 
tified, for the QELS 15, 21. However there is certainly a connection between the VFAM and QELS, which can be found if we consider the interpretation of the QELS15,21, and the time resolved o-Ps annihilation 22. Novikov showed that the origin of the QELS can be the thirdorder anharmonic term in the vibrational Hamiltonian², so that the QELS is proportional to the square of the Grüneisen coefficient. From another point of view, the same author and collaborators 15 showed theoretically that the QELS is proportional to $\Delta F_{h}$, by adding to the harmonic potential a term of interaction between the vibration and the fluctuation of the dynamic hole volume or of the free volume. If the Hamiltonian in ref.21 does not depend necessarily on the presence of dynamic holes, the term of interaction, between the hole volume fluctuation and the vibration, originates from third order terms (enhanced by the dynamic holes) in the vibrational Hamiltonian. In the model of Novikove1, the fact, that the inverse of the characteristic time corresponds approximately to one third of the boson peak frequency, is justified because the anharmonic third order term is relatively strong for the modes in the boson peak.

The inverse time, $1 / \tau_{1}$, corresponds to a frequency of $0.5 \mathrm{THz}(\sim 2 \mathrm{meV})$, that is very close to the frequency of the boson peak $(1.65 \mathrm{meV})^{14}$, and not to one third. This shows that the VFAM is not identical to the QELS, and can not be interpreted simply by the anharmonic third-order terms in the vibrational Hamiltonian, and by the model of Novikov2 2 . The presence of a dynamic hole close to a vibrating polymeric nanodomain does not only damp, but can probably, in given situations depending upon the hole volume, the vibration frequency or the position of the hole relatively the vibrating domain, overdamp the lowfrequency vibrational modes. In the case of overdamping, the relation between the decay time $\tau$ and the frequency $\nu$ of the vibration is $2 \pi \nu \tau=1$. With $\tau=\tau_{1}$, the frequency $\nu(0.5 \mathrm{THz})$ is slightly higher than the one of the boson peak $(0.4 \mathrm{THz})$. The suggested interpretation is, therefore, that the VFAM corresponds to the low-frequency modes in the boson peak, which are overdamped by nearby dynamic holes. This is in agreement with the proportionality between $\rho$ and $\Delta F_{h}$ (Figure-5), and the Q-independence of $\tau_{1}$ or the localization. This interpretation is confirmed by the time resolved o-Ps annihilation22. Vass 
et al. showed that, for an organic glass forming liquid, the thermal behavior of the ratio $I_{3} / I_{1}$ ( $I_{1}$ and $I_{3}$ being the intensities of respectively the fast p-Ps and the slow o-Ps annihilations) can be understood if very fast molecular motions (characteristic time less than $1 \mathrm{ps)} \mathrm{make}$ the dynamic hole volume to fluctuate and, then, the o-Ps, that is trapped in the hole, to annihilate with a non negligible probability22. Finally, the dynamic holes or free volumes have two effects, on the one hand, they enhance the effect of the anharmonic third order terms in the potential, and they also possibly overdamp the vibration of neighboring molecular groups. At this point, it must be noticed that very recently Schmidt et al.2. effect of the hole volume on the QELS, because they observed that the QELS of PMMA did not depend very much on the density, which varied from a sample to another by the pressure applied during the cooling of the polymer. However, it was carefully verified that, from $\mathrm{T}=100 \mathrm{~K}$ up to $T_{g}$, the QELS is proportional to the dynamic hole volume, and not to the total hole volument. Now, it is likely that the pressure applied during the cooling has principally an effect on the static hole volume, on which the QELS does not depend.

The time $\tau_{1}$ is weakly dependent on temperature. However, its slight decrease with temperature seems unavoidable for a good fit of the experimental decays (Figure-3). If, a priori, it is surprising to observe a relaxation time, that decreases with temperature, this thermal behavior of $\tau_{1}$ is consistent with the proposed model of overdamping. As recalled above, from a temperature of $100 \mathrm{~K}$, there is a clear softening of the low-frequency vibrational modes. The characteristic time $\tau_{1}$ is inversly proportional to the vibration frequency $\nu$ ( $\tau_{1} \approx$ $1 / 2 \pi \nu)$, and therefore one expects that $\tau_{1}$ decreases with temperature, as it is experimentally observed (Figure-3). The effect of the dynamic holes on the fast dynamics, which are the apparent result of a slow relaxation, would deserve a more extensive study. It is likely that the softening of the low-frequency modes is due to the presence of the dynamical holes.

One can be surprised to find a so short relaxation time, or, in other words, that vibrational modes of a frequency, that is equal to $0.5 \mathrm{THz}$, are overdamped at $T<T_{g}$. This can be justified by the relatively large volume fraction of dynamical holes in PC (Figure-5). On the other hand, similar relaxation times were obtained in other glasses like the ortho-terphenyl, 
respectively, by depolarized light scattering 7 , and by Brillouin scattering $\mathrm{G}$.

The limited resolution of the IN6 neutron spectrometer, in spite of its deconvolution from the experimental dynamical structure factor, does not allow to be confident of $S_{n h}(Q, t)$ at times longer than $10 \mathrm{ps}$, so that the determined value of the $\tau_{2}$ relaxation time is rather imprecise. However some properties of the slower relaxation, at which all the glass participates, contrary to the very fast relaxation, are clear enough: $\tau_{2}$ is temperature and momentum transfer dependent (Table-1), like the fast relaxation observed by Floudas et al.24. From Figure-6, an activation energy of about $10.5 \mathrm{~kJ} /$ mole can be deduced. $1 / \tau_{2}$ increases clearly with Q, but the experimental precision does not allow to give a quantitative Q-dependence. As a matter of fact, $\tau_{2}$ is an effective relaxation time, which accounts certainly for several different molecular motions. The activation energy for the methyl-group rotation is 15-21 $\mathrm{kJ} / \mathrm{mol} 1 \mathrm{17}$, as noted above, and $14.6 \mathrm{~kJ} / \mathrm{mole}$ for the segmental -C-C-rotational motion 2 . Both activation energies are close to that deduced for $\tau_{2}$ (Figure-6), so that it is likely that $\tau_{2}$

accounts for the methyl-group rotation and the segmental relaxation. The Q-dependence of the effective time $\tau_{2}$ may indicate that the relaxation is partially diffusion-like on short molecular distances, and would correspond to the short-time regime of the segmental relaxation $\mathrm{g}$.

\section{CONCLUSION}

The experimental neutron scattering study brings new informations about the anharmonic motions in a polymeric glass at $T<T_{g}$. The anharmonic motions appear from a low temperature $(50 \mathrm{~K})$ in comparison with $T_{g}(420 \mathrm{~K})$. A very fast relaxation characterized by a time close to $0.3 \mathrm{ps}$, that is momentum transfer independent and decreases very slightly with temperature, is observed at temperatures higher than $130 \mathrm{~K}$. This is interpreted by the overdamping of the low-frequency vibrational modes, that are responsible for the boson peak. The identical thermal behavior for the proportion of the glass, that is submitted to the very fast anharmonic motion, and for the dynamical hole volume fraction, shows that the overdamping of the low-frequency modes is due to the presence of the dynamical holes 
in the neighboring of the vibrating nanodomain.

\section{ACKNOWLEDGEMENTS}

The authors are very grateful to K. Ngai and V.N. Novikov for valuable discussions and suggestions. 


\section{REFERENCES}

${ }^{1}$ V. Arrighi, J. S. Higgins, A. N. Burgess and W. S. Howells, Macromolecules28 2745 (1995)

${ }^{2}$ N. V. Surovtsev, A. Mermet, E. Duval and V. N. Novikov, J. Chem. Phys. 1046818 (1996)

${ }^{3}$ A. Mermet, E. Duval, N. V. Surovtsev, J. F. Jal, A. J. Dianoux and A. F. Yee, Europhys. Lett. 38515 (1997)

${ }^{4}$ E. Duval, A. Mermet, N. Surovtsev, J. F. Jal and A. J. Dianoux, Phil. Mag. B 77457 (1998)

${ }^{5}$ E. Duval, A. Mermet, N. V. Surovtsev and A. J. Dianoux, J. Non-Cryst. solids 203-207 235 (1998)

${ }^{6}$ V. N. Novikov, N. V. Surovtsev, E. Duval and A. Mermet, Europhys. Lett. 40165 (1997)

${ }^{7}$ A. Patkowski, W. Steffen, G. Meier and E. W. Fisher, J. Non-Cryst. Solids 172-174 52 (1994)

${ }^{8}$ G. Monaco, D. Fioretto, C. Masciovecchio, G. Ruocco and F. Sette, Phys. Rev. Lett. 82 $1776(1999)$

${ }^{9}$ J. Colmenero and A. Arbe, Phys. Rev. B 5713508 (1998)

10 J. Colmenero, A. Arbe and A. Alegria, Phys. Rev. Lett. 712603 (1993)

${ }^{11}$ A. Fontana, F. Rocca, M. P. Fontana, B. Rossi and A. J. Dianoux, Phys. Rev. B 413778 (1990)

12 B. Frick andD. Richter, Phys. Rev. B 4714795 (1993)

${ }^{13}$ J. Wuttke, Phys. Rev. E to be published (2000)

${ }^{14}$ L. Saviot, E. Duval, N. Surovtsev, J. F. Jal and A. J. Dianoux, Phys. Rev. B 6018 (1999) 
${ }^{15}$ V. N. Novikov, A. P. Sokolov, B. Strube, N. V. Surovtsev, E. Duval and A. Mermet, J. Chem. Phys. 1071057 (1997)

${ }^{16}$ V. N. Novikov, private communication

${ }^{17}$ C. Schmidt, K. J. Kuhn and H. W. Spiess, Progr. Colloid and Polymer Sci. 7171 (1985)

${ }^{18}$ M. Wehrle, G. P. Hellmann and H. W. Spiess, Colloid and Polymer Sci. 265815 (1987)

${ }^{19}$ M. Bée, in Quasielastic Neutron Scattering, Ed. Adam Hilger (1988)

${ }^{20}$ H. A. Hristov, B. Bolan, A. F. Yee, L. Xie and D. W. Gidley, Macromolecules 298507 (1996)

${ }^{21}$ V. N. Novikov, Phys. Rev. B 588367 (1998)

${ }^{22}$ Sz. Vass, A. Patkowski, E. W. Fisher, K. Süvegh and A. Vértes, Europhys. Lett. 46815 (1999)

${ }^{23}$ M. Schmidt, A. Brodin, P. Jacobsson and F. H. J. Maurer, J. Chem. Phys. 1121020 (2000)

${ }^{24}$ G. Floudas, J. S. Higgins, G. Meier, F. Kremer and E. W. Fischer, Macromolecules 26 $1676(1993)$

${ }^{25}$ M. Hutnik, A. S. Argon and U. W. Suter, Macromolecules 245970 (1991) 


\section{FIGURES}

FIG. 1. Mean square displacement $\left\langle u^{2}\right\rangle$ plotted against the temperature.

FIG. 2. Debye-Waller corrected $S(Q, \omega)$ scattering spectra. (a) for $Q=1.9 \AA^{-1}$, at different temperatures: $15 \mathrm{~K}$ (full line), $30 \mathrm{~K}$ (triangles), $110 \mathrm{~K}$ (circles), $220 \mathrm{~K}$ (squares), $300 \mathrm{~K}$ (stars). (b) at $\mathrm{T}=390 \mathrm{~K}$, for different momentum transfers: $\mathrm{Q}=0.91 \AA^{-1}$ (squares), $1.33 \AA^{-1}$ (stars), 1.9 $\stackrel{\circ}{A}^{-1}$.

FIG. 3. Normalized scattering function from non-harmonic or relaxational motions, for $Q=1.9 \AA^{-1}$, at different temperatures: $390 \mathrm{~K}$ (plus), $340 \mathrm{~K}$ (crosses), $300 \mathrm{~K}$ (stars), $260 \mathrm{~K}$ (open squares), $220 \mathrm{~K}$ (full squares), 170K (open circles). The decays are fitted with the expression 3.2, using the parameters given in Table-1. The insert shows the decays, that are obtained by the Fourier transform, from 0 ps to 2 ps in linear coordinates, with the fits (full lines).

FIG. 4. Normalized scattering function from non-harmonic or relaxational motions, at $T=390 K$, for different momentum transfers: $\mathrm{Q}=1.9 \AA^{-1}$ (plus), $1.62 \AA^{-1}$ (crosses), $1.33 \AA^{-1}$ (stars), $1.11 \AA^{-1}$ (open squares), $0.91 \AA^{-1}$ (full squares). The decays are fitted with the expression 3.2, using the parameters given in Table-1. The insert shows the decays from 0 ps to 2 ps in linear coordinates.

FIG. 5. The ratio $\rho$ of the very fast relaxing part is plotted against the dynamic hole volume fraction $\Delta F_{h}$. The temperatures corresponding to the different points are: $170 \mathrm{~K}, 220 \mathrm{~K}, 260 \mathrm{~K}$, 300K, 340K, 390K.

FIG. 6. The inverse time $1 / \tau_{2}$ is plotted against the inverse of temperature for $Q=1.9 \AA^{-1}$. The dotted line corresponds to an activation energy of $2.5 \mathrm{kcal} / \mathrm{mole}$. 


\section{TABLES}

TABLE I. Parameters in 3.2 deduced from the fits in Figures 3 and 4

\begin{tabular}{ccccc}
\hline \hline $\mathrm{Q}\left(\AA^{-1}\right)$ & $\mathrm{T}(\mathrm{K})$ & $\rho$ & $\tau_{1}(p s)$ & $\tau_{2}(p s)$ \\
\hline 1.9 & 170 & 0.18 & 0.22 & $\infty$ \\
1.9 & 220 & 0.25 & 0.24 & 634 \\
1.9 & 260 & 0.31 & 0.25 & 163 \\
1.9 & 300 & 0.37 & 0.28 & 100 \\
1.9 & 340 & 0.43 & 0.30 & 60 \\
1.9 & 390 & 0.51 & 0.33 & 87 \\
1.62 & 390 & 0.43 & 0.33 & 129 \\
1.33 & 390 & 0.32 & 0.33 & 149 \\
1.1 & 390 & 0.27 & 0.31 & 162 \\
0.91 & 390 & 0.24 & 0.27 & \\
\hline \hline
\end{tabular}




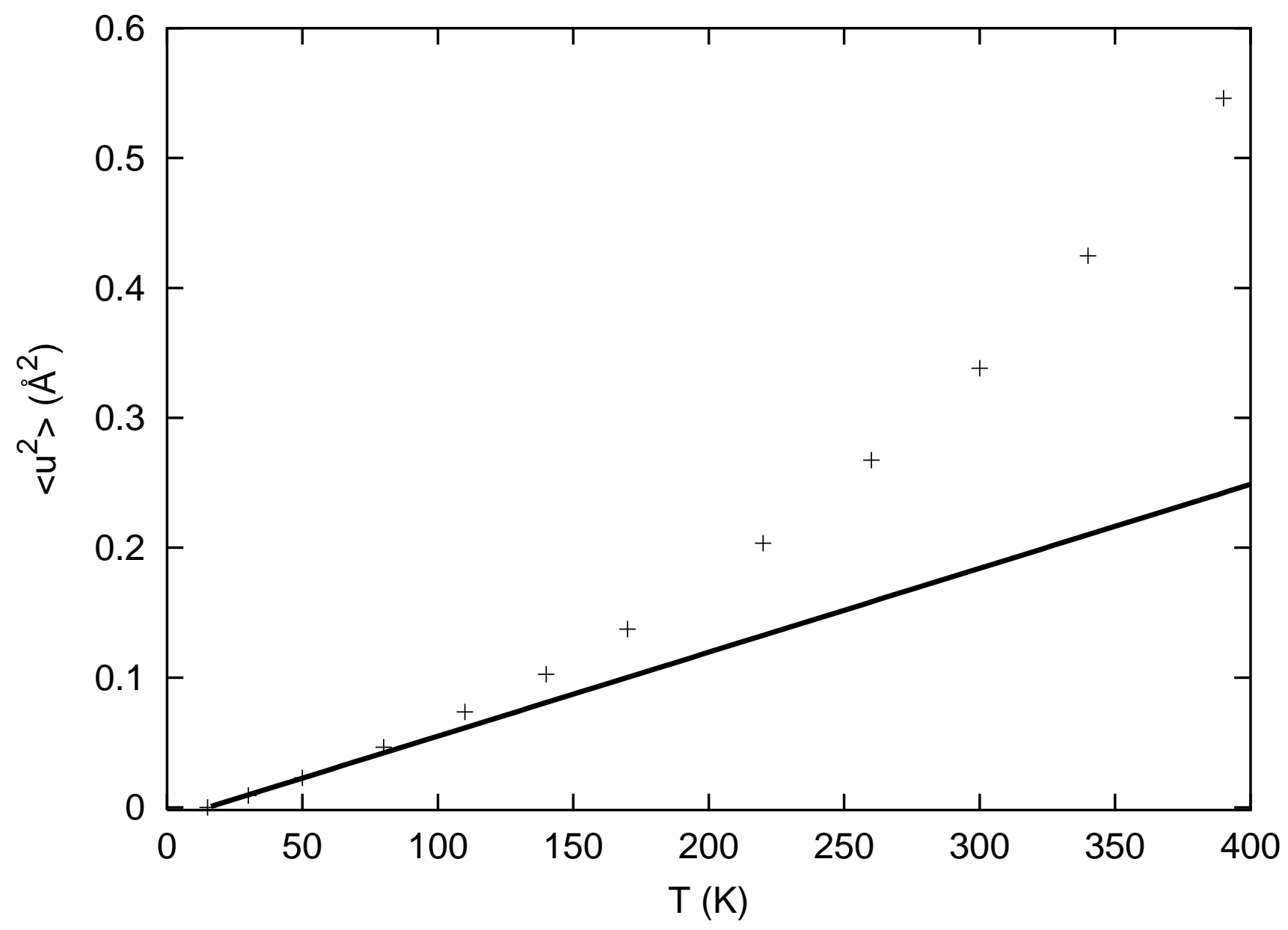




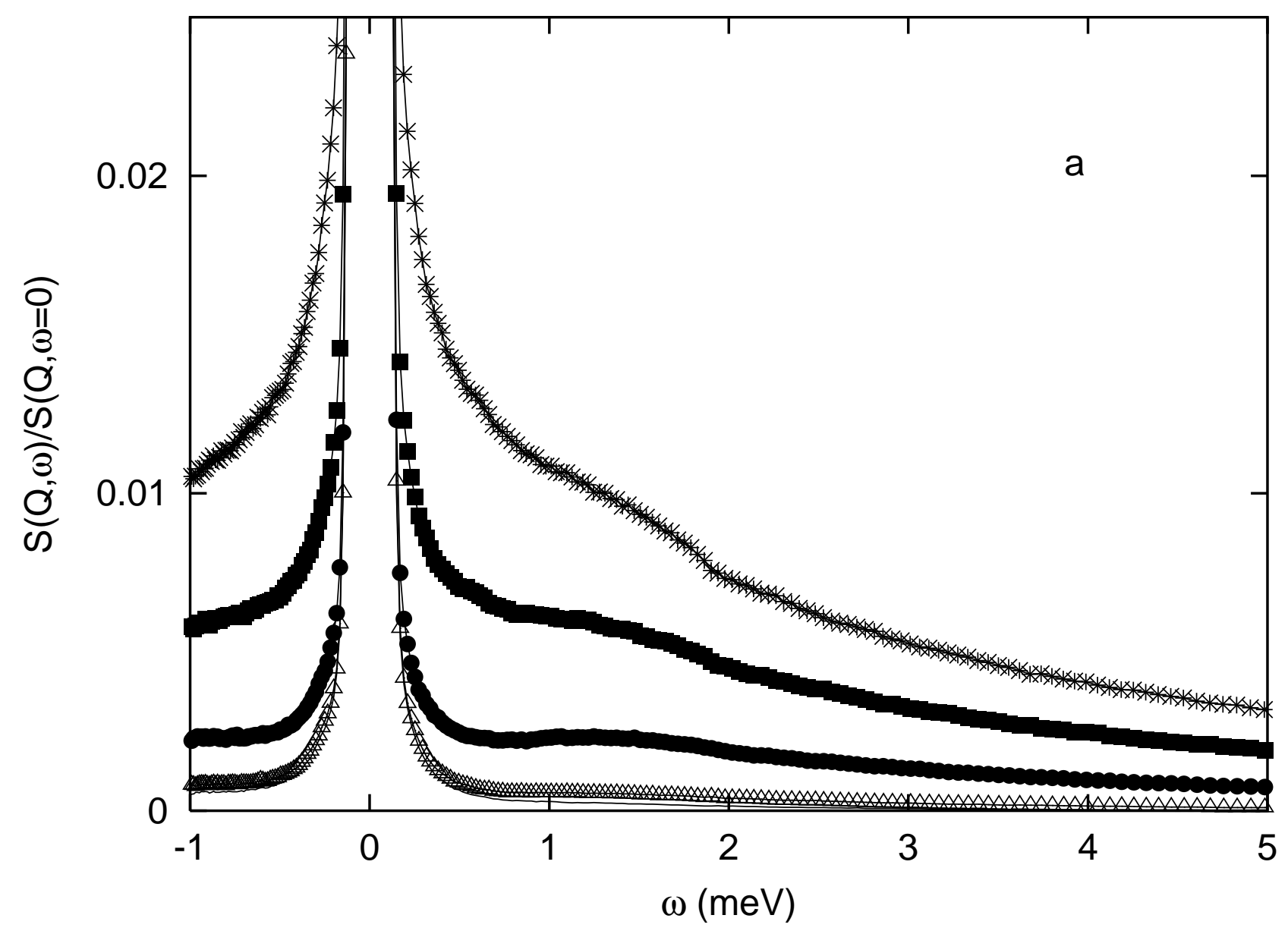




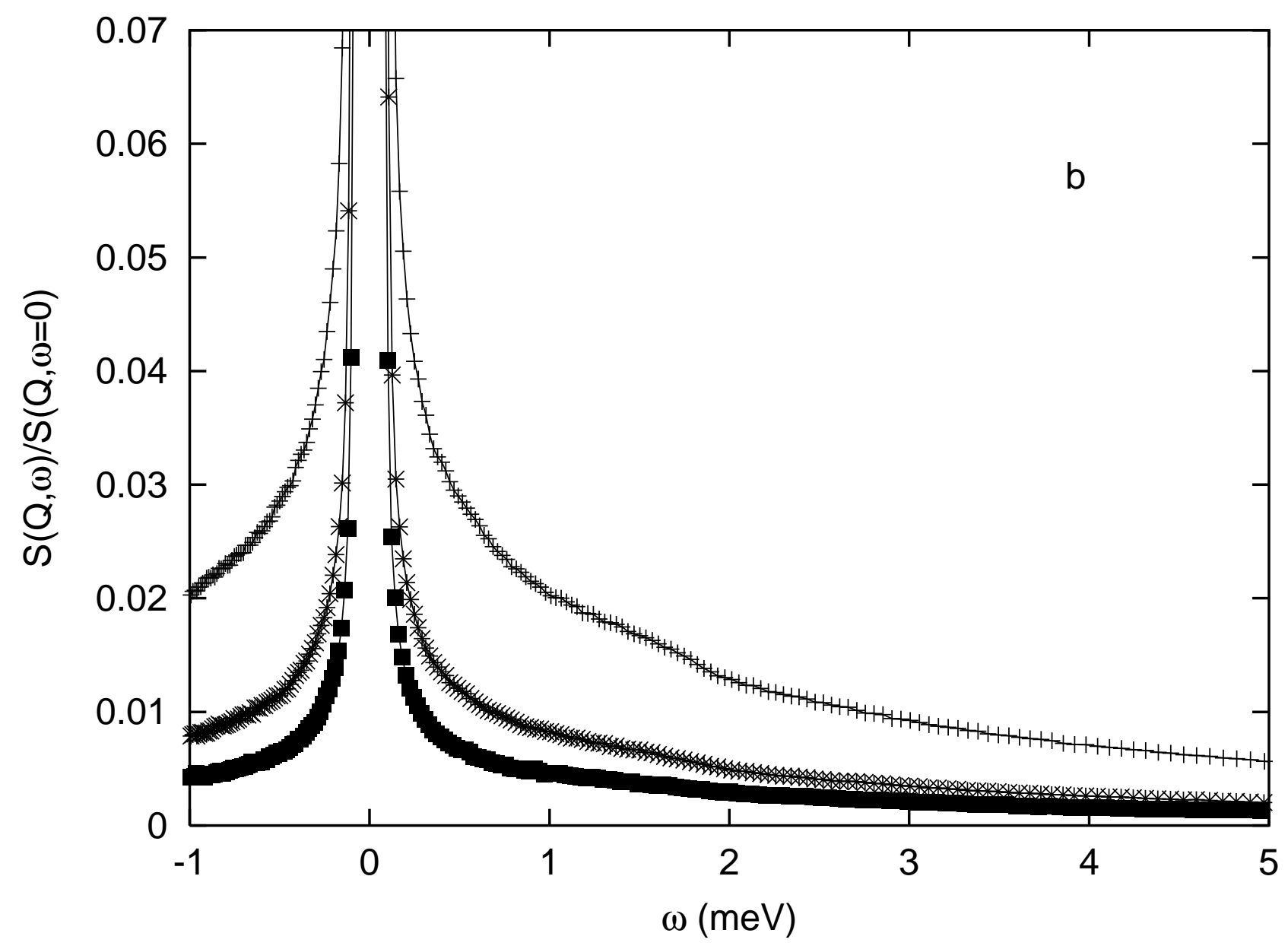




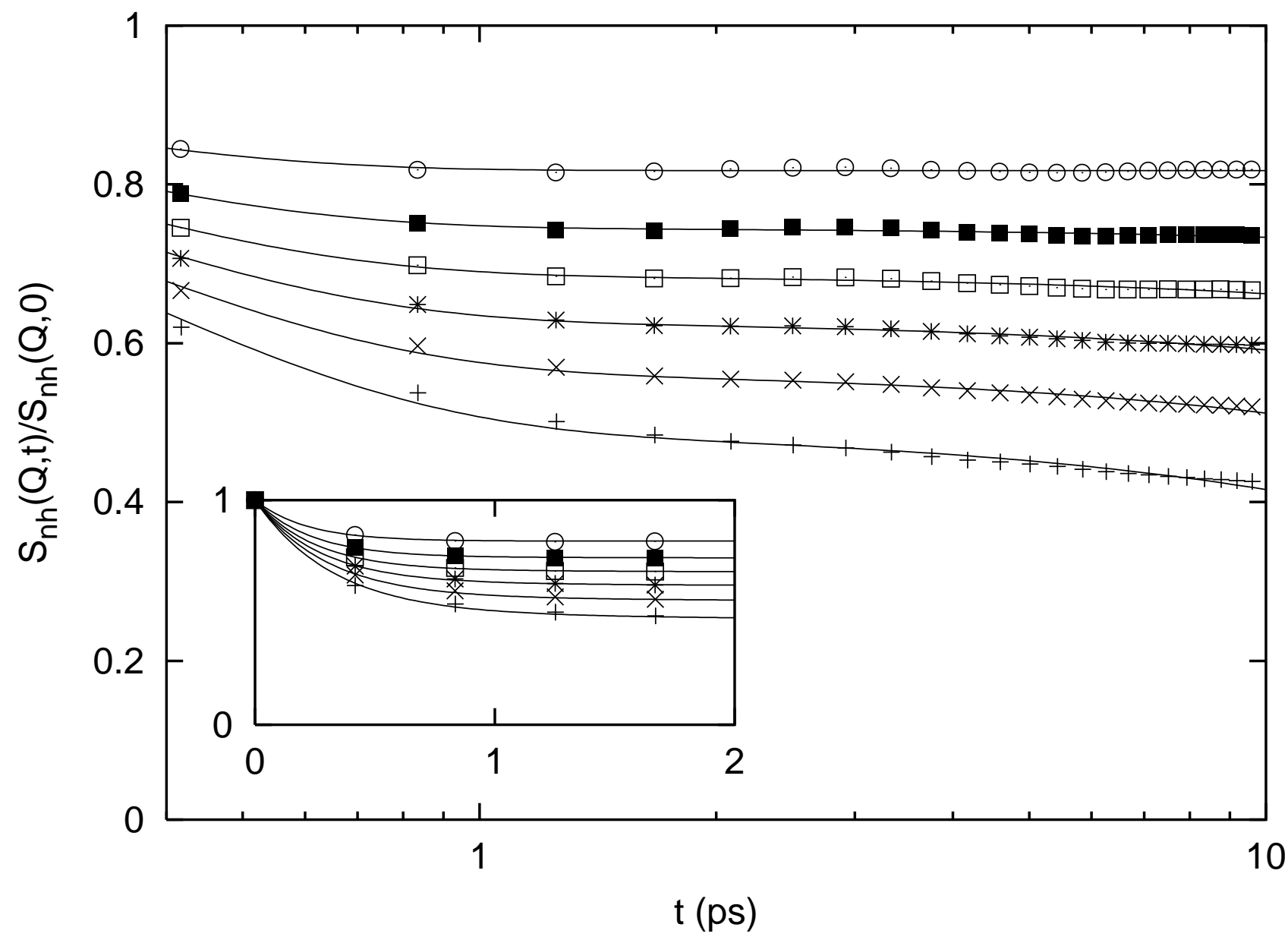




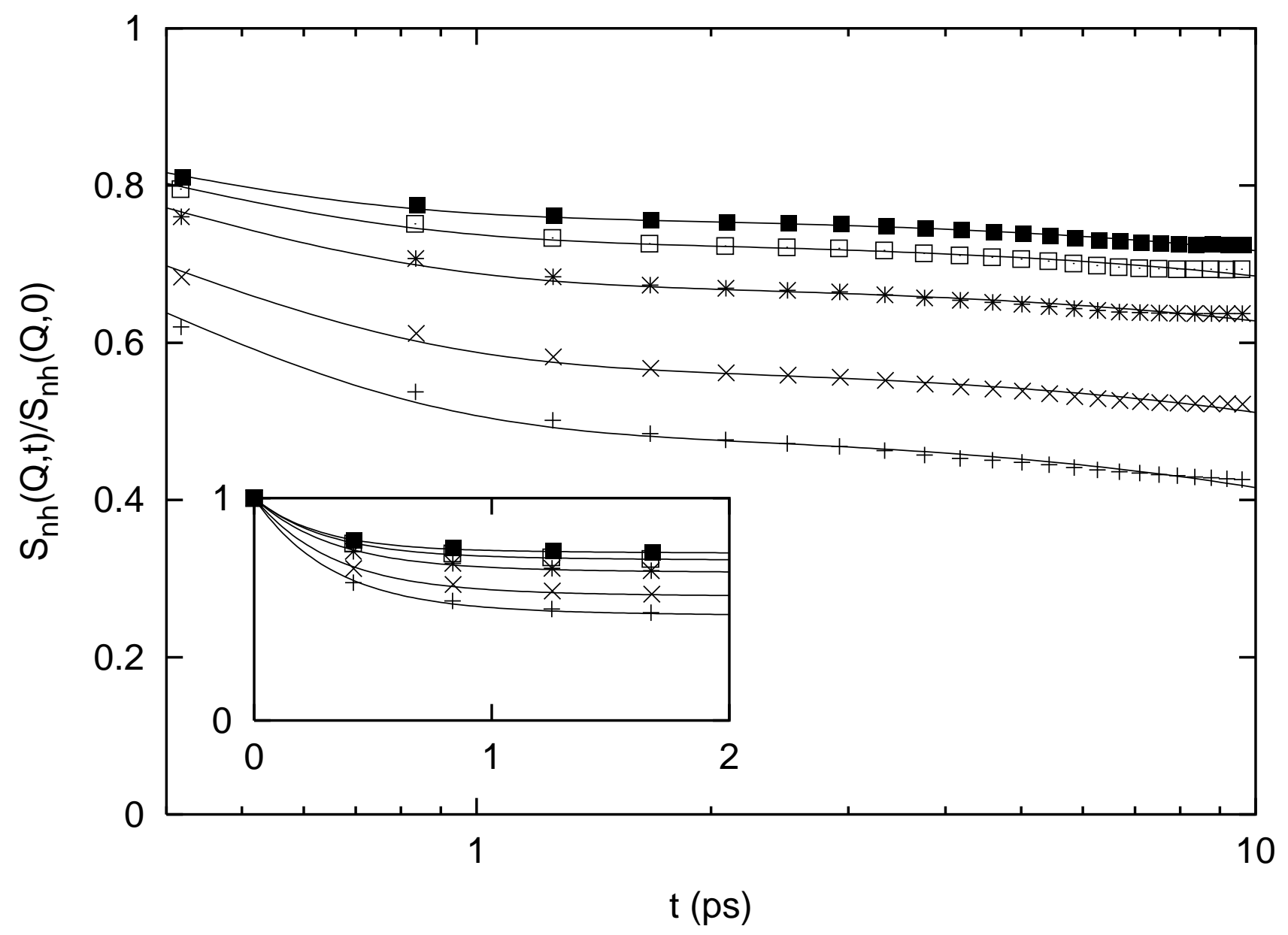




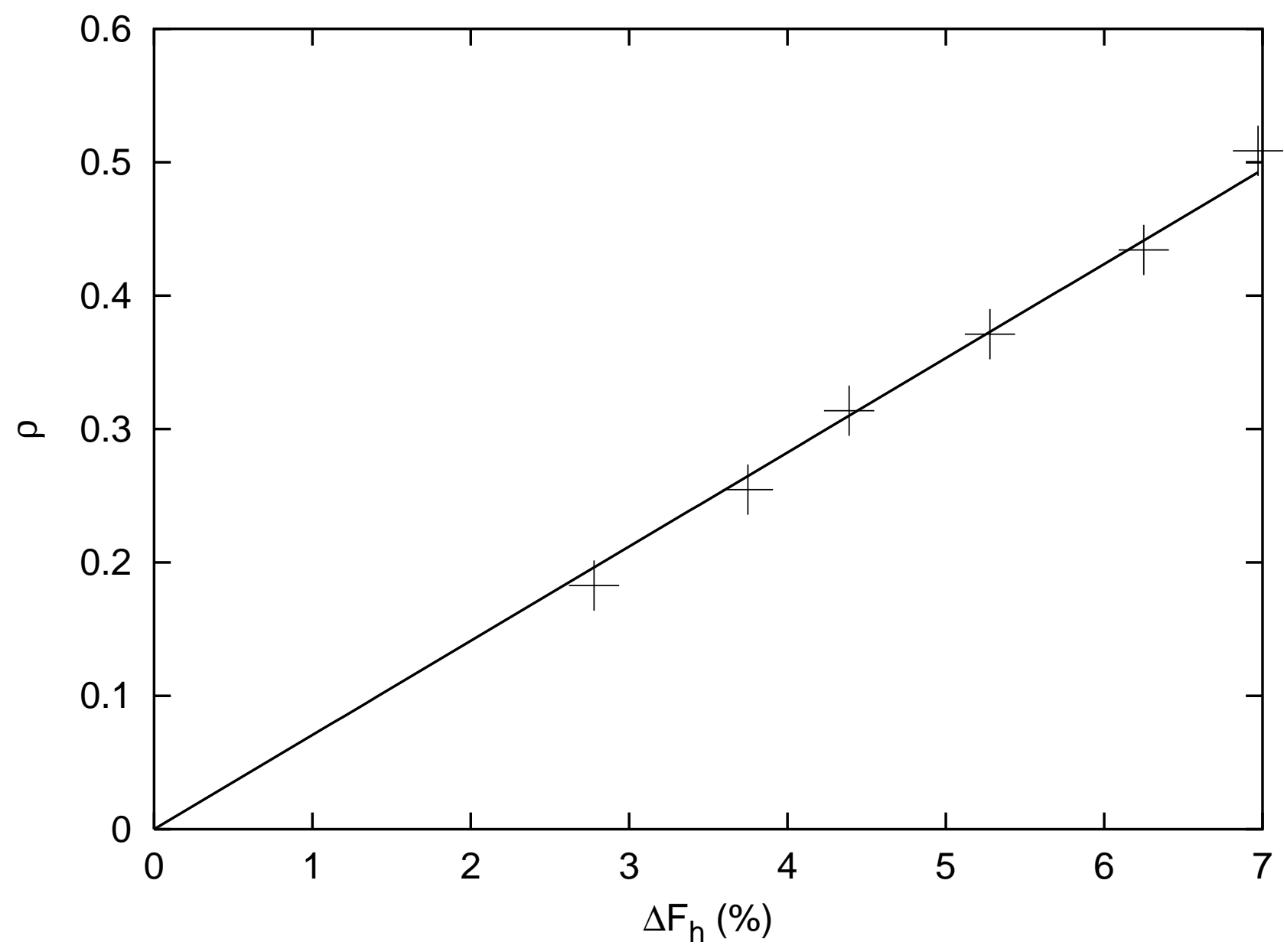




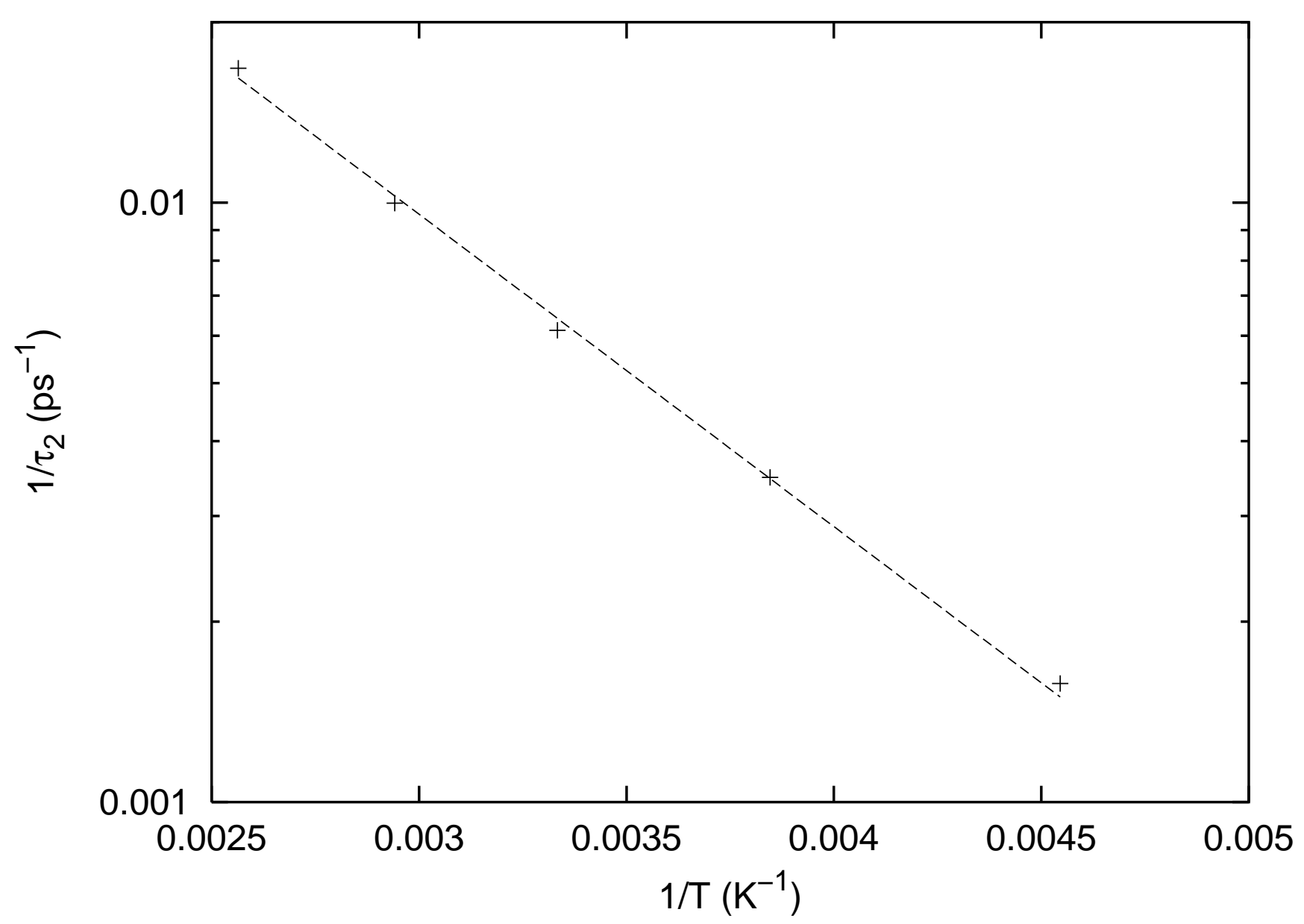

